\title{
Shortening the Acclimation and Degradation Lag of Xenobiotics by Enriching the Energy Content of Microbial Populations
}

\author{
Huong Lan Nguyen ${ }^{1}$, Nyuk-Min Chong ${ }^{2}$, Ha Manh Bui ${ }^{3 *}$ \\ ${ }^{1}$ Faculty of Biotechnology and Environmental Engineering, Hochiminh City University of Food Industry, \\ Ho Chi Minh City, Vietnam \\ ${ }^{2}$ Department of Environmental Engineering, Dayeh University, Changhua, Taiwan \\ ${ }^{3}$ Department of Environmental Sciences, Saigon University, Ho Chi Minh City, Vietnam
}

Received: 9 October 2017

Accepted: 10 December 2017

\begin{abstract}
We studied shortening the acclimation and enhancement of the degradation rate for a xenobiotic organic pollutant using microbial populations grown in suspension as activated sludge. Soil populations cultivated as raw activated sludge in a steady-state fed-batch reactor and the raw sludge were re-cultured (energy enriched) with biogenic substrates and compared for their performances in acclimation and degradation of 2,4-D. The cells' mass and ATP content were also measured during the 2,4-D acclimation and degradation. The optimal concentration of supplemental biogenic substrate and most suitable time point of re-cultivation that could produce the most shortening of lag phase were determined in a previous study. The purpose of this study was to find the true mechanism of this shortening based on the contents of cell internal ATP. As compared with the raw sludge, the enriched sludge contained a higher amount of energy at the start of acclimation and brought a shortened lag time during the acclimation process. External energy (biogenic substrates's energy) invested into activated sludge cells could improve the microorganisms in acclimation to a xenobiotic by sustaining the energy needed for the difficult xenobiotic metabolism.
\end{abstract}

Keywords: xenobiotic, biodegradation, activated sludge, acclimation, cell ATP

\section{Introduction}

Xenobiotic organic compounds are mostly man-made and are comprised of chlorinated ring structures that make them difficult to degrade by natural or indigenous microorganisms [1]. Indigenous microorganisms are usually degrade xenobiotics through novel enzymatic

*e-mail: manhhakg@sgu.edu.vn mechanisms during the acclimation process [2]. The common weakness for indigenous populations (such as activated sludge) to acclimate to xenobiotics is the prolonged lag time that would lengthen the overall time required for total degradation of the xenobiotic. Even after the sludge populations had acquired degradability from acclimation, xenobiotic organic compounds are hard for microorganisms to break down due to their stable structure [3-4]. In addition to the energy consumptive acclimation and the subsequent stable structure breaking processes, 
initial catabolism steps of oxidative 2,4-D degradation also consume reducing power [5-6] that reduces energy generation at the stage of oxidative phosphorylation. As a result, the management of cells' energy budget becomes another difficulty that the cells must overcome, in addition to the evolution of the degradation pathway. The manifold of degradation difficulties are disadvantageous for activated sludge microorganisms in their further treatment of xenobiotic pollutants that may be encountered in soil and groundwater remediation and also in wastewater treatment systems. Degradation of 2,4-D with glucose using a mixed culture consisting of 3-4 dominant Pseudomonas sp. by Lackmann et al. [7] showed that the rates of 2,4-D degradation were the same as those observed when the medium did not contain glucose, whereas the rate of glucose utilization was lower than that observed in the absence of 2,4-D. The author's conclusion was that 2,4-D inhibits glucose removal while glucose does not affect 2,4-D biodegradation. To ease such an energy issue for the xenobiotic degrading microorganisms, external biogenic substrates can be fed to growing microorganisms so that the metabolism of biogenic substrate would enrich the cells' energy reserve before the cells start to tackle the xenobiotic target. In addition, substrate-level metabolism of biogenic substrate may also bring NADH enrichment, which can be resourceful for ATP formation. Previous studies have shown that the biodegradation rate of some xenobiotics can be enhanced by adding biogenic organics such as glucose and peptone on pentachlorophenol degradation [8]. Also, enhancing nutrients such as nitrogen, phosphate, and minerals were found to be beneficial for xenobiotic degradation [9]. The mentioned enhancement effect was found in the microbial population that exits in suspended growth, such as the activated sludge [10], and in soil matrix [6]. However, the reasons for enhancement of xenobiotic degradation with adding biogenic substrates reported in the above literature were seldom, if any, related to the energy aspect of the degrader cells during their acclimation and/or degradation processes. This paper presents a logistical examination of the true mechanism by which biogenic substrates are beneficial to xenobiotic degradation, based on bioenergetics of xenobiotic metabolism.

Growth on biogenic substrates is logically known to furnish energy to microbial cells. However, the energy obtained from biogenic metabolism is suspected to be foreign to that obtained from the in-line metabolism of a xenobiotic. In other words, whether such externally added energy content is useful revenue for xenobiotic degraders to have a definite improvement of their xenobiotic degradation rate is not clear presently. The purpose of this study, therefore, was to investigate the possibility that a higher initial energy content in microbial cells is able to remedy the energy deficit and to increase cell yield, and thereby shorten acclimation lag phase and enhance degradation rate for a model xenobiotic. To fulfil the purpose of this study, indigenous microbial populations cultivated as activated sludge were harvested directly (raw) from a steady-state growing reactor, and such sludge re-cultivated with a feed of biogenic substrates to enrich its energy contents were tested in parallel to compare the performances in acclimation and degradation for the xenobiotic between both sludge. Time courses of acclimation and degradation of the xenobiotic, biomass growth, and ATP contents in both the raw and re-cultivated sludge biomasses were measured and compared. The results thus obtained provide novel answers to unknowns pertaining to the bioenergetics aspect of xenobiotic degradation.

\section{Experimental}

\section{Xenobiotic and Sludge Biomass}

The target xenobiotic was the herbicide 2,4-dichlorophenoxyacetic acid (2,4-D) supplied by Merck (Germany). The initial microbial seeds were obtained from a soil that had neither a record of 2,4-D nor metal contamination. This indigenous seed was grown in a fed-batch reactor that was refed once daily with a fresh medium containing biogenic substrates (120 mg/L sucrose and $50 \mathrm{mg} / \mathrm{L}$ peptone) and minerals: $\mathrm{FeCl}_{3} 1.0 \mathrm{mg} / \mathrm{L}, \mathrm{NH}_{4} \mathrm{Cl} 30.0 \mathrm{mg} / \mathrm{L}, \mathrm{K}_{2} \mathrm{HPO}_{4} 200.0 \mathrm{mg} / \mathrm{L}$, $\mathrm{KH}_{2} \mathrm{PO}_{4} 156.0 \mathrm{mg} / \mathrm{L}, \mathrm{MgSO}_{4} .7 \mathrm{H}_{2} \mathrm{O} 65.0 \mathrm{mg} / \mathrm{L}$, and $\mathrm{pH}=7.8$. The biomass obtained from this fed-batch reactor is referred to as the raw sludge. The raw sludge grew at a slow rate under a mean cell residence time $(q \mathrm{c})$ of 10 days, which must be equivalent to the late stationary phase of growth in the batch system.

The raw sludge described above was enriched with ATP content by feeding with biogenic substrates of sucrose and peptone (the resulting sludge was referred to as the re-cultivated or enriched sludge). The enrichmentcultivation media contained $100 \mathrm{mg} / \mathrm{L}$ of sucrose and $25 \mathrm{mg} / \mathrm{L}$ of peptone, plus the minerals listed above, in multiple reactors that were operated in shake-flasks. In the enrichment cultivation, biogenic feeds were administered once approximately every 20 hours prior to the start of acclimation tests. After this cultivation the enriched sludge, which was tested to make sure that its ATP content was higher than that of raw sludge, was harvested and used in new reactors for the 2,4-D acclimation and degradation tests.

\section{Acclimation and Degradation Tests}

The sludge biomasses from the 2 cultivation schemes described above (namely raw and re-cultivated sludge) were used for acclimation and degradation of 2,4-D. The starting sludge concentrations (SS) were measured, and calculated amounts were added to the acclimation reactors to make a target $100 \mathrm{mgSS} / \mathrm{L}$ therein. Media in acclimation reactors contained $100 \mathrm{mg} / \mathrm{L}$ of 2,4-D and the minerals listed above.

Acclimation and degradation of 2,4-D were conducted in batch shake-flasks $(300 \mathrm{~mL}$ conical flasks containing $200 \mathrm{~mL}$ liquid, shaken at $100 \mathrm{rpm}$ orbital, at room 
temperature of $\left.25 \pm 2^{\circ} \mathrm{C}\right)$. Samples were withdrawn at regular intervals (typically one day) from the flasks for the measurements of 2,4-D concentrations remaining in solution, concentrations that indicated biomass growth, and ATP contained in the cells. The acclimation and degradation experiments were conducted in triplicate.

\section{Analytical Methods}

Soluble 2,4-D concentrations in the degradation reactor suspensions were measured with HPLC (Shimadzu 20AT). Concentrations of sludge biomass were measured as the dried weight (SS) of the biomasses from the filterable (Whatman $\mathrm{GF} / \mathrm{C}$ ) portion of reactor suspensions. SS and COD were analysed following the standard methods for the examination of water and wastewater [11].

ATP contained in suspended microbial cells was measured along every course of 2,4-D acclimation and degradation. Suspensions of known SS were harvested from an acclimation and degradation shake-flask for ATP extraction. Detailed procedures for extracting ATP from sludge cells can be found from our previous publication [12]. ATP was extracted from an aliquot of sludge ( $\eta \mathrm{ml}$, with $\kappa \mathrm{mg} / \mathrm{L}$ of SS). The sludge first must be cleaned with $10 \mathrm{ml}$ of $0.005 \%$ sodium dodecyl sulfate (SDS) and then centrifuged for $10 \mathrm{~min}$ at $5,000 \mathrm{rpm}$ under $4^{\circ} \mathrm{C}$. Endogenous (internal) ATP contained in the centrifuged sludge cells was determined. The pellet was washed by putting in $10 \mathrm{~mL}$ deionized water and then centrifuged for $10 \mathrm{~min}$ at $5,000 \mathrm{rpm}$ at $4^{\circ} \mathrm{C}$. The bacterial cells were collected from the pellet and then resuspended using $3 \mathrm{ml}$ of TCA in Tris buffer: $0.02 \mathrm{M}$ Tris and 2\% TCA $\left(\mathrm{pH} 7.8\right.$, temperature $22 \pm 1^{\circ} \mathrm{C}$ ). The resulting suspension was poured to a $7 \mathrm{~mL}$ bead blasting tube with $0.5 \mathrm{~g}$ of $0.5 \mathrm{~mm}$ sterile glass beads, after which the wall of the Falcol tube was rinsed with $0.5 \mathrm{~mL}$ (accurate) of the extraction solution and the rinsate was added to the same bead blasting tube. All cell suspensions that were treated with above extraction agents were bead-blasted using Minilys (Bertin Technologies, France). Blasting was run at $4,000 \mathrm{rpm}$ for 30 seconds, followed by one min of incubation on ice, after which the suspension was disrupted again for 30 seconds. The whole suspension containing the extract and glass beads and debris was then transferred to a $15 \mathrm{~mL}$ Falcon tube. The wall and cap of the bead blasting tube were cleaned with $0.5 \mathrm{~mL}$ (accurate) Tris buffer and transferred completely into the $15 \mathrm{~mL}$ Falcon tube followed by centrifuging at 5,000 rpm for $5 \mathrm{~min} .4 .0 \mathrm{~mL}$ of supernatant containing ATP extracted from the cells was filtered through a $0.22 \mu \mathrm{m}$ membrane filter (Millex-GS) to remove suspended particles.

Then these extracts were measured on a Shimadzu HPLC system equipped with a UV Detector at $254 \mathrm{~nm}$ (Model20AT). About $5 \mu$ lof analyte solution was injected into an HPLC valve using a Puradisc $25 \mathrm{~mm}$ (Sterile and Endotoxin Free $0.2 \mu \mathrm{m}$ PES Filter Media) and Terumo Syringe $(5 \mathrm{ml})$ at room temperature $\left(25 \pm 2^{\circ} \mathrm{C}\right)$. The needed analysis compounds were separated on a Phenomenex
Luna reverse phase (RP-18) column, $250 \mathrm{~mm} \times 4.6 \mathrm{~mm}$ packed with $\mathrm{C} 18$ stationary phase with particle size $5 \mu \mathrm{m}$.

The ATP concentrations $(\chi \mu \mathrm{g}-\mathrm{ATP} / \mathrm{mL})$ were identified by comparing retention time of chromatographic peak analysis with those of reference compounds. ATP stored in the sludge was calculated as $\mu \mathrm{g}$-ATP $\mathrm{mg} / \mathrm{SS}$ with the equation $(\chi \mu \mathrm{gATP} / \mathrm{mL} \times 4.0 \mathrm{~mL}) /\left(\eta \times 10^{-3} \mathrm{~L} \times\right.$ $\kappa \mathrm{mgSS} / \mathrm{L})$, where $\chi$ is ATP concentration in an extract; $\kappa$ and $\eta$ are, respectively, SS concentration and volume $(\mathrm{mL})$ of activated sludge sample from which ATP was extracted; and $4.0 \mathrm{~mL}$ is the final volume of supernatant (in Falcon tube) containing ATP extracted from the activated sludge cells.

\section{Results and Discussion}

Fig. 1 shows the courses of 2,4-D degradation, the growth of sludge biomass (in SS), and internal ATP contents of sludge cells for the sludge that was harvested raw from a slowing growing reactor. Fig. 1a) indicates that the lag of raw sludge acclimation to 2,4-D was approximately 4 days and was followed with a fast degradation rate that is typical of activated sludge degradation of 2,4-D. SS growth produced a yield of $0.25 \mathrm{mgSS} / \mathrm{gCOD}$ from 2,4-D. Degradation experiments of this study were conducted in fully aerated reactors such that oxidative metabolism pathways prevailed. Depletion of 2,4-D shown in the time-curves in all figures indicated full degradation of 2,4-D, without undetectable accumulation of organic intermediates [13].

As a comparison to the performance of raw sludge, Fig. 2 shows the change of 2,4-D concentrations, SS
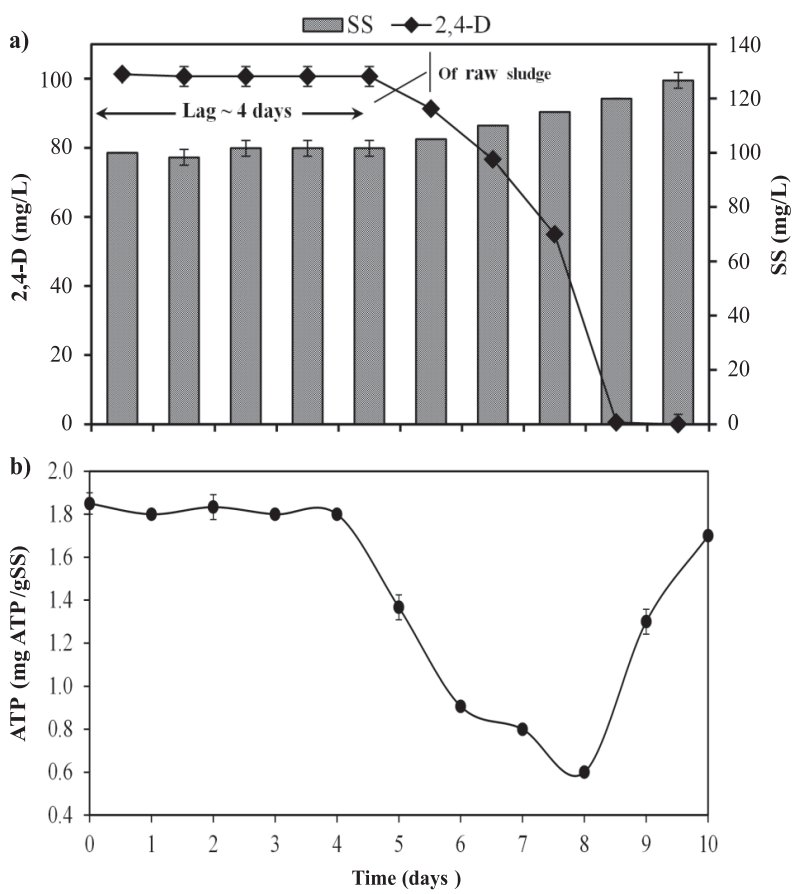

Fig. 1. Time courses of a) 2,4-D, SS and b) ATP contents of raw sludge in acclimation and degradation of 2,4-D. 

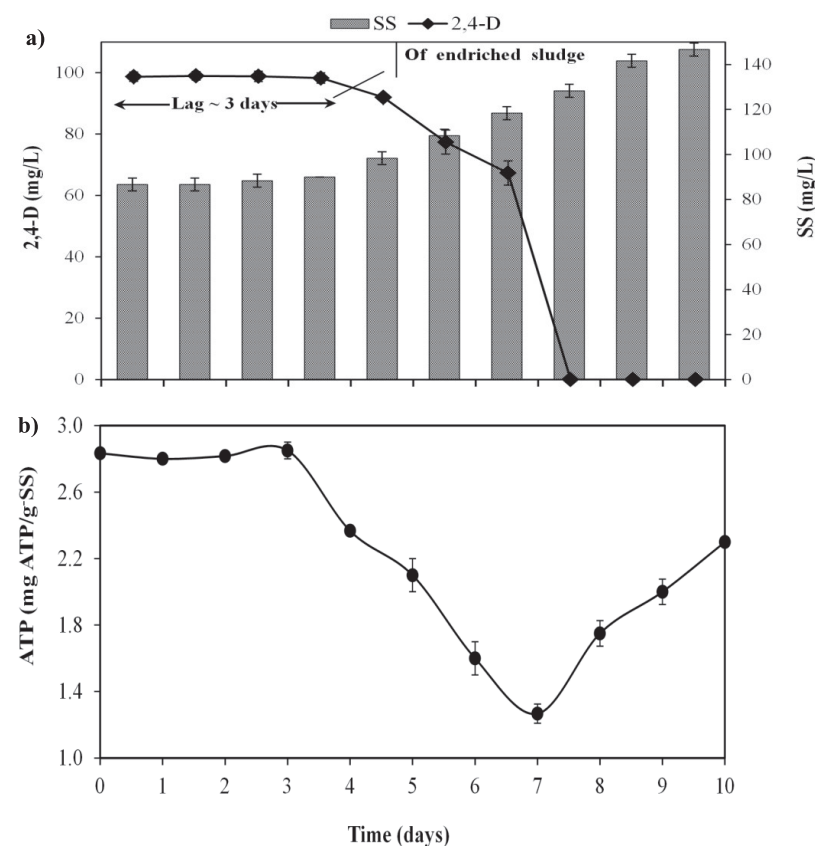

Fig. 2. Time courses of a) 2,4-D, SS and b) ATP contents of recultivated sludge in acclimation and degradation of 2,4-D.

growth, and internal ATP contents during xenobiotic acclimation and degradation of the re-cultivated and energy-enriched sludge. Fig. 2a) shows that after the sludge cells were re-cultivated with biogenic substrate and had its initial energy content increased, the cells would acclimate to 2,4-D with a lag time shortened by approximately one day compared to that of raw sludge.

Fig. 3 shows that the enriched sludge had a higher internal ATP content than raw sludge (2.56 and $1.85 \mathrm{mgATP} / \mathrm{gSS}$, respectively) at the start of 2,4D acclimation. At the time of completion of 2,4D degradation when internal ATP deficit was the maximum, a slightly more internal ATP was consumed by the cells of the enriched sludge. The yield of re-cultivated activated sludge growing on 2,4-D was found to be slightly but noticeably higher than that of raw sludge ( 0.34 and 0.25 gcell $/ \mathrm{gCOD}$, respectively).

Comparing Figs 1 and 2, the enriched sludge made an acclimation lag time of approximately one day shorter

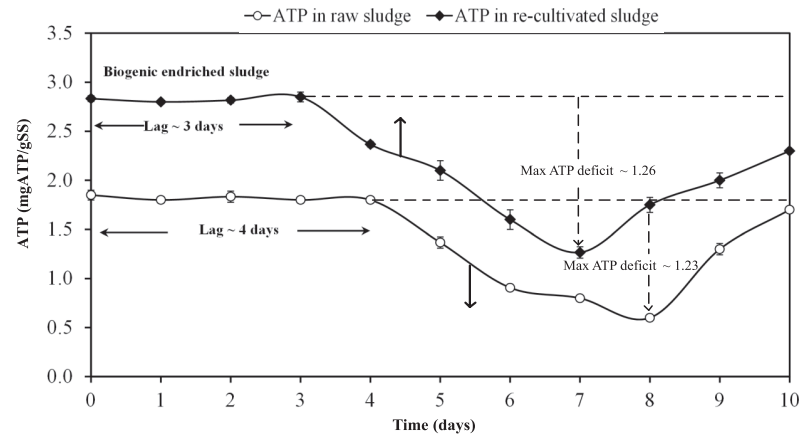

Fig. 3. Differences between ATP contents in raw and re-cultivated sludge in acclimation and degradation of 2,4-D. than that of the raw sludge. Degradation rates after acclimation of both types of sludge were not significantly different. Although the acclimation phase was apparently not ATP intensive, shortening the acclimation lag can be viewed as an earlier start of degradation (see Figs 1a) and 2a) comparisons of degradation curvatures). The more energy-rich re-cultivated sludge could more readily provide the energy demand to start degradation.

Yield of both types of sludge were low compared to that from sludge growth on common biogenic substrates. A low yield of microbial biomass in metabolizing a xenobiotic is due to the diversion of energy available from substrate metabolism to satisfy the energy requirement for breaking the stable xenobiotic structure or biotransformation (e.g., reductive dichlorination) prior to suitable catabolism. With a chlorinated xenobiotic such as 2,4-D, reducing power in the form of NADH may also be spent in dechlorination in an oxygenated reaction and thus reduces the production of ATP. Fig. 1b) shows the course of ATP contents in sludge cells during their acclimation and degradation of 2,4-D. In the acclimation phase, ATP in unit cell mass was essentially constant. Evolution of degradative enzyme is shown to be mildly energy expensive. During the degradation phase, however, ATP was lowered noticeably corresponding to the disappearance of 2,4-D. The enriched sludge had a higher initial ATP content than raw sludge (Fig. 3), and when substrate 2,4-D was completely degraded, slightly more ATP was consumed to have a higher yield. A higher cell yield for the energy-enriched cells can be most reasonably explained by the provision of more energy for cell yield. This finding is also indicative of the efficiency of microbial cells in the allocation of available energy to balance between total cell yield and ATP reserve of individual cells. The maximum ATP deficit (Figure 3) for the raw sludge may have been bound by the lowest limit of ATP content in the cell. The enriched sludge, on the other hand, still had not spent ATP to this limit so that some more energy can be channeled to cell mass yield.

The yields of activated sludge on 2,4-D is low due to microbial cells breaking down specific bonds, especially de-chlorination, that consumes reducing power (NADH) [3]. In the process of 2,4-D metabolism, microbial populations must reproduce new cells. Under limiting ATP generating sources, the cost of energy for cell yield is deducted from the ATP reserve of the cells. The finding that ATP per cell deceases after 2,4-D degradation indicates how microorganisms could set the priority for survival: a metabolism strategy by which new cell production enjoys a higher priority to energy richness in the cells. Regaining ATP per cell occurred immediately at 2,4-D depletion (after the 8th day). The initial regain of ATP can be attributed to growth on biogenic downstream products. At a time much later than when 2,4-D was completely depleted, continuous regain of ATP per cell is most possibly provided by the internal food storage of each cell or by the decay of some cells that could happen in an act to keep healthy survivor cells with fuller energy reserve. How microbial cells manage their energy budget, 
especially about how they attain eventual ATP contents, is an important topic requiring further investigation that must feature the balances of all aspects of bioenergetic requirements.

To summarize, it is observed that a microbial population must spend energy to degrade a xenobiotic and as a result an energy deficit occurs in microbial cells after degrading the xenobiotic. Sometimes the microorganisms could obtain energy by degrading some xenobiotic compounds. Growing microorganisms on biogenic substrates at a time point prior to xenobiotic degradation increases the initial ATP contents in the cells and this energy was useful in increasing or compensating part of the ATP that may be spent in metabolism of the xenobiotic. This external energy enrichment brings to the xenobiotic degradation system the benefit of acclimation lag shortening, and thus the earlier completion of xenobiotic degradation. Enriching microorganism cells' energy with a feed of biogenic substrates prior to its encounter with a xenobiotic is a feasible technique for improving the remediation and treatment performance of microorganisms for xenobiotic pollutants.

\section{Conclusions}

When microorganisms are put in contact with xenobiotic compounds, in a curious environment, acclimation to these compounds may occur. By comparing the internal ATP contents of raw and enriched activated sludge cells, shortenings of acclimation lag phase and enhancement of xenobiotic degradation rate can be found to result from the amount of energy-activated sludge cells contained prior to the acclimation reaction. The required energy can be invested into the sludge cells by growing the cells with biogenic substrates that were fed to the cells at a most suitable concentration and at a point prior to the acclimation reaction. Shortening lag time and enhancing the overall rate of 2,4-D degradation by enriching ATP contents of cells provides practicing environmental engineers a viable strategy in enhancing efficacy of xenobiotic treatment in a wastewater treatment plant.

\section{Acknowledgements}

This study was partially supported by a research grant MOST 103-2221-E-212-001-MY3 provided by the Ministry of Science and Technology of Taiwan, the Republic of China. Parts of the contents of this manuscript were presented at an AWSPT'16 conference of International ASET Inc. (international-aset.com), which permits this contribution for journal publication.

\section{References}

1. NIKEL P.I., PÉREZ-PANTOJA D., DE LORENZO V. Why are chlorinated pollutants so difficult to degrade aerobically? Redox stress limits 1,3-dichloprop-1-ene metabolism by Pseudomonas pavonaceae. Phil. Trans. R. Soc. B., 368 (1616), 20120377, 2013.

2. KUMAR B.L., GOPAL D.V.R.S. Effective role of indigenous microorganisms for sustainable environment. 3 Biotech., 5 (6), 867, 2015.

3. CHONG N.M., TSAI S.C., LE T.N. The biomass yielding process of xenobiotic degradation. Bioresour. Technol., 101 (12), 4337, 2010.

4. YANG Z., XU X., DAI M., WANG L., SHI X., GUO R. Rapid degradation of 2,4-dichlorophenoxyacetic acid facilitated by acetate under methanogenic condition. Bioresour. Technol., 232, 146, 2017.

5. KASBERG T., KASCHABEK S., MÜLLER D., REINEKE W. Maleylacetate reductases functioning in the degradation of chloroaromatics. Int. Biodeterior. Biodegradation, 37 (34), 247, 1996.

6. MIR Z.A., ALI S., TYAGI A., ALI A., BHAT J.A., JAISWAL P., QARI H.A., OVES M. Degradation and conversion of endosulfan by newly isolated Pseudomonas mendocina ZAM1 strain. 3 Biotech., 7 (3), 211, 2017.

7. LACKMANN R.K., MAIER W.J., SHAMAT N. Removal of chlorinated organics by coventional biological waste treatment, Presented at: Proceedings of the Thirty-Fifth Industrial Waste Conference, pp. 502-515, 1980.

8. PATEL B.P., KUMAR A. Multi-substrate biodegradation of chlorophenols by defined microbial consortium. 3 Biotech., 6 (2), 191, 2016.

9. ABURTO-MEDINA A., BALL A.S. Microorganisms involved in anaerobic benzene degradation. Ann. Microbiol., 65 (3), 1201, 2015.

10. CHONG N.M., LUONG M., HWU C.S. Biogenic substrate benefits activated sludge in acclimation to a xenobiotic. Bioresour. Technol., 104, 181, 2012.

11. RICE E.W., BRIDGEWATER L. Standard Methods for the Examination of Water and Wastewater, 20th Edition, APHA American Public Health Association, pp. 531-545, 2012.

12. NGUYEN L.H., CHONG N.M. Development of an ATP measurement method suitable for xenobiotic treatment activated sludge biomass. J. Chromatogr. B Biomed. Sci. Appl., 1000, 69, 2015.

13. CHONG N.M., CHANG C.-S., TSAI S.C. Evolutions of microbial degradation pathways for parent xenobiotic and for its metabolites follow different schemes. Environ. Sci. Pollut. R., 19 (8), 3276, 2012. 